\title{
EVALUACIÓN DE UNA ESTRATEGIA CENTRALIZADA Y UNA DESCENTRALIZADA PARA EL CONTROL DE CONTAMINACIÓN EN CUERPOS HÍDRICOS
}

\author{
EVALUATION OF A CENTRALIZED AND A DECENTRALIZED \\ STRATEGY FOR THE CONTROL OF POLLUTION IN WATER BODIES
}

\author{
Ángela Vanessa Polanía Janzasoy ${ }^{1}$ \\ Luisa Fernanda Calderón-Vallejo ${ }^{2}$ \\ ${ }^{1}$ Est. Master of Science Programme in Water Resources and Environmental Management - \\ Leibniz Universität Hannover. Facultad de Ingeniería, Universidad del Valle \\ 2 M.Sc., Universidad Nacional Abierta y a Distancia UNAD - \\ Escuela de Ciencias Agrícolas, Pecuarias y del Medio Ambiente (ECAPMA). \\ 1 angela.polania@correounivalle.edu.co \\ 2 luisa.calderon@unad.edu.co
}

\section{Resumen}

El objetivo de este trabajo fue la construcción y aplicación de una estrategia hipotética (descentralizada) que contempla tres pasos: 1) maximizar la prevención o reducir la producción de agua residual, 2) tratar para la reutilización y 3) aprovechar la capacidad de autodepuración del cuerpo hídrico y compararla con una estrategia convencional (centralizada) fundamentada en la implementación de Plantas de Tratamiento de Agua Residual-PTARs como única estrategia para reducir la carga de contaminación entrante a cuerpos hídricos específicamente en la cuenca alta del río Cauca, la cual es la segunda cuenca hidrográfica más importante de Colombia, y contribuir así al planteamiento de la recuperación del río, en el tramo comprendido entre La Balsa y Anacaro (389 km aproximadamente). Es importante mencionar que no se conocen estudios en el contexto que analicen la zona de estudio con la implementación de estas estrategias que van en armonía con la Gestión Integrada del Recurso Hídrico-GIRH, razón por la cual su estudio y análisis se convierte en un aspecto importante para planear a futuro el mejoramiento de la calidad de agua de los cuerpos hídricos receptores.

Los resultados de la investigación mostraron el potencial de la estrategia de los tres pasos que, combinado con la priorización, logran mayores remociones de carga contaminante en comparación con la estrategia centralizada. La carga total obtenida respectivamente fue $64,65 \mathrm{~T}$ $\mathrm{DBO}_{5} * \mathrm{~d}^{-1}$ y $86,34 \mathrm{~T} \mathrm{DBO}_{5} * \mathrm{~d}^{-1}$, partiendo de una línea base en el 2011 de 169,54T $\mathrm{DBO}_{5} * \mathrm{~d}^{-1}$. 
logrando reducciones de $63 \%$ y $54 \%$, respectivamente. Por otro lado, el OD mínimo en el río Cauca conseguido fue $3,5 \mathrm{mg}^{*} \mathrm{~L}^{-1}$ para la alternativa no convencional (descentralizada) y $2,5 \mathrm{mg}^{*} \mathrm{~L}^{-1}$ para la alternativa centralizada, siendo la primer estrategia planteada mayor contribuyente a la recuperación del río Cauca en su cuenca alta.

Palabras claves: aplicación de estrategias de descontaminación, control de contaminación, reducción de consumo, reúso, río Cauca, tratamiento de aguas residuales.

\section{Abstract}

The aim of this study was the construction and application of a hypothetical (decentralized) strategy that includes three steps: 1) maximize the prevention or reduce the production of wastewater, 2) treat for reuse and 3) validation of self-purification capacity of the water body and compare it with a conventional (centralized) strategy based on the implementation of Wastewater Treatment Plants-WWTPs as the only strategy to reduce the load of incoming contamination to water bodies specifically in the upper Cauca River basin, which is the second most important river basin in Colombia, and thus contribute to the approach in the recovery of the river, in the stretch between La Balsa and Anacaro (around $389 \mathrm{~km}$ ). It is important to mention that there are no known studies in the context that analyze the study area with the implementation of these strategies that are in harmony with the Integrated Management of Water Resources-IWRM, which is why its study and analysis becomes an important aspect to plan in the future the improvement of the water quality of the receiving water bodies. The results of the investigation demonstrated the potential of the three-step strategy combined with the prioritization achieved greater removals of pollutant load compared to the centralized strategy, the total load was $64,65 \mathrm{DBO}_{5} \mathrm{~T}^{*} \mathrm{~d}^{-1}$ and $86,34 \mathrm{DBO}_{5} \mathrm{~T}^{*} \mathrm{~d}^{-1}$, respectively; starting from a baseline (2011) of $169.54 \mathrm{DBO}_{5} \mathrm{~T}^{*} \mathrm{~d}^{-1}$, reaching reductions of $63 \%$ and $54 \%$, respectively. On the other hand, the minimum OD in the Cauca River achieved $3,5 \mathrm{mg}^{*} \mathrm{~L}^{-1}$ for the unconventional (decentralized) alternative and $2,5 \mathrm{mg}^{*} \mathrm{~L}^{-1}$ for the conventional alternative, with the first strategy being the largest contributor to the recovery of the Cauca River in its upper basin.

Keywords: Application of decontamination strategies, Cauca River, consumption reduction, pollution control, reuse, wastewater treatment plant.

\begin{tabular}{|c|c|}
\hline CINARA & $\begin{array}{l}\text { Instituto de Investigación y Desarrollo en Abastecimiento de Agua, } \\
\text { Saneamiento Ambiental y Conservación del Recurso Hídrico }\end{array}$ \\
\hline CRC & Corporación Autónoma Regional del Cauca \\
\hline CVC & Corporación Autónoma Regional del Valle del Cauca \\
\hline DBO_5 & Demanda Biológica de Oxígeno, medida transcurridos cinco días de reacción \\
\hline EMCALI & Empresas Municipales de Cali \\
\hline GIRH & Gestión Integrada del Recurso Hídrico \\
\hline OD & Oxígeno Disuelto \\
\hline PML & Producción más Limpia \\
\hline PTAR & Planta de Tratamiento de Aguas Residuales \\
\hline SSPD & Superintendencia de Servicios Públicos Domiciliarios \\
\hline
\end{tabular}




\section{Introducción}

A nivel mundial, el deterioro de la calidad del recurso hídrico se agudiza a través del tiempo debido al incremento de la población y al desarrollo limitado de planes de saneamiento básico que promuevan el uso de tecnologías limpias para asegurar la permanencia de los usos actuales hasta las generaciones futuras. Es por ello que la comunidad internacional, desde mediados del siglo $\mathrm{XX}$, sitúa la problemática del agua como una prioridad, realizando diversos foros mundiales donde se han concertado criterios y estrategias para el mejoramiento del recurso hídrico a nivel mundial (Polanía J., 2014).

Entre los conceptos que surgieron entorno a la adecuada GIRH, se destaca el desarrollado por los autores Nhapi y Gijzen (2005) quienes proponen un modelo integral, que considera como sistema de análisis, desde la vivienda hasta el cuerpo hídrico receptor de la contaminación. La metodología se denomina los Tres Pasos y consiste en: 1) reducción drástica del consumo de agua potable y la generación de aguas residuales 2) implementar tecnologías de tratamiento sostenibles, con procesos eficientes, en donde se optimice la calidad de sus efluentes para hacerlos aptos para el reúso, al igual que otros subproductos del tratamiento como el biogás y los biosólidos; y 3) aprovechar la capacidad de autodepuración del cuerpo de agua.

El presente estudio de caso hipotético, se desarrolló en el marco de la estrategia de los Tres Pasos, y tuvo como objetivo principal plantear un escenario en el que se compara una estrategia de control de la contaminación descentralizada, fomentando el reúso lo más cerca posible a la fuente que lo genera (considerando condiciones locales y normativas) y compararlo con las tecnologías centralizadas como lo son las comúnmente concebidas y empleadas PTARs municipales.
A lo largo del manuscrito se presentan los elementos que se tuvieron en cuenta para la construcción de cada estrategia tomando como punto de partida estudios previos en los que ha participado el Instituto Cinara de la Universidad del Valle de Cali - Colombia, además de información brindada por las autoridades ambientales y empresas proveedoras de los servicios públicos vinculadas con la zona de estudio. Posteriormente se presenta un análisis comparativo de los sistemas centralizado y descentralizado propuestos. Se toma como línea base el año 2011 dado que es aquel con mayores estudios de calidad de agua reportados para el río Cauca.

\section{Antecedentes}

Un sistema descentralizado puede consistir en sistemas de tratamiento individuales en el sitio o en combinación con instalaciones centralizadas. El grado de colectivización en cualquier etapa de los procesos de tratamiento y reutilización o dispersión estará determinado por una variedad de circunstancias locales, que incluyen la topografía, las características del sitio y del suelo, la densidad y el tipo de desarrollo, los deseos de la comunidad con respecto al uso de la tierra y sitios de reutilización potencial y / o sitios donde la descarga sería permisible (Chirisa et al., 2017).

Los sistemas descentralizados pueden ser tan efectivos como los centralizados, especialmente cuando se aplican en zonas de expansión dentro del marco del desarrollo urbano planeado (Dou et al., 2017). Sin embargo, en las ciudades pequeñas, la cantidad de aguas residuales generadas es muy inferior, lo que resulta en alcantarillado de un tamaño más pequeño y un menor costo de bombeo. Además, el suelo está disponible abundantemente, por lo que se prefiere en estos casos el tratamiento centralizado (Gupta, 2016). 
Los sistemas municipales de tratamiento de aguas residuales en América Latina son actualmente insostenibles ya que se caracterizan por tratar elevadas cantidades de agua, generando asimismo un elevado costo en el transporte de agua residual afuera de las ciudades en donde usualmente están localizados los sistemas centralizados (Chirisa et al., 2017).

Los recursos hídricos de Colombia se encuentran altamente contaminados por el vertimiento desmedido y sin control de aguas residuales domésticas a estos. En la actualidad, las soluciones de tipo convencional son las más comunes de implementar, tal es el caso de la construcción de PTARs, las cuales en nuestro país no funcionan adecuadamente y además son inversiones costosas en algunos casos según los niveles de tratamiento que sean requeridos. Según el informe técnico sobre sistemas de tratamiento de aguas residuales en Colombia de la SSPD del año 2013, solo el $26 \%$ de los municipios del país cuenta con algún sistema de tratamiento de las aguas residuales y tan solo el $18.5 \%$ de estos sistemas incluyen tratamiento de tipo secundario sin llegar en todos los casos a alcanzar las eficiencias de diseño (SSPDb, 2013).

Dado este contexto, se hace necesario estudiar otro tipo de estrategias que permitan contribuir con la recuperación de los recursos hídricos como se explica a continuación.

\section{Metodología Área de estudio}

La zona de estudio considerada fue la cuenca alta del río Cauca en el tramo La Balsa-Anacaro, tiene una longitud aproximada de $389 \mathrm{~km}$, con un descenso del nivel 1.020 al 901m.s.n.m. El tramo del río Cauca entre las estaciones La Balsa y Anacaro, incluye la zona de influencia en el departamento del Valle del Cauca (jurisdicción de la CVC) y del
Departamento del Cauca (jurisdicción de la CRC). La Estación la Balsa, está ubicada $65 \mathrm{~km}$ aguas debajo del Embalse de Salvajina, el cual entró en operación en 1985 y fue concebido con fines multipropósito (generación de energía, regulación de caudales, abastecimiento y recreación). A lo largo del río están ubicadas 15 estaciones de monitoreo que han proporcionado información de cantidad y de calidad del recurso, desde hace más de 50 años (Sandoval \& Ramírez, 2007), de los cuales para este caso solo se usó caudal, $\mathrm{DBO}_{5}$ y OD por ser indicadores específicos del nivel de contaminación en el río y capacidad de asimilación de la corriente. (ver en los anexos figura 1)

El río Cauca, en su cuenca alta en el tramo La Salvajina - Anacaro, tiene 29 subcuencas que drenan hacia el río, los mayores usos del agua en el tramo son: abastecimiento humano (es la fuente abastecedora de alrededor 2 millones (al año 2012) de habitantes de la ciudad de Cali), recreación, actividad agropecuaria, en especial para la caña de azúcar y otros cultivos semestrales, minería (principalmente explotación de materiales de arrastre), navegación de bajo calado; receptor de vertimientos generados en las distintas actividades de tipo económico y doméstico de los alcantarillados de la mayoría de los municipios vecinos a su cauce. En total son 44 municipios que descargan directamente a la cuenca alta del río Cauca. Adicionalmente, este recibe los vertimientos generados por las industrias establecidas en el norte del departamento del Cauca, la cual cuenta con la presencia de tres ingenios azucareros, fábricas de producción de Papel, fábrica de alimentos y bebidas y factorías livianas debido a la ley 218 de 1994 o Ley Páez (mediante incentivos tributarios se promovía al sector industrial, invertir en dicha zona) y el corredor industrial Cali - Yumbo (Sandoval \& Ramírez, 2007; Moreno, 2014, Polanía J., 2014). 


\section{Línea base (2011)}

A partir de los diagnósticos de Planes de Saneamiento y Manejo de Vertimientos (PSMV), los resultados de diferentes proyectos desarrollados por el Grupo de investigación GIRH del Instituto CINARA de la Universidad del Valle, incluyendo proyectos de investigación (Zorrilla, 2012), tesis (Suárez, 2010; Zambrano, 2012; Jaramillo, 2014; Urrego, 2014), se obtuvieron datos históricos sobre la cantidad y calidad del agua del río Cauca y los ríos tributarios, suministrados por CVC (2013) y CRC (2010 y 2013) con los cuales se realizó el presente estudio.

De igual manera para complementar la información secundaria obtenida, se consultó al sector privado (Sánchez, 2013) y al sector público (EMCALI, 2012; SSPD, 2010 y 2013a) sobre el estado y conformación de las PTARs de la región. Con esta información y el último trabajo de modelación del río Cauca desarrollado por investigadores del Instituto Cinara con el modelo Qual2K versión libre 2.07 (Urrego, 2014), se construyó la línea base de cantidad y calidad del agua río Cauca y sus principales tributarios para la condición de verano (la más crítica), definiéndose como año base, el 2011. Esta información, sirvió como punto de partida para estimar las cargas y los caudales de las estrategias analizadas, la convencional (centralizada) y la no convencional (descentralizada). Se definió el año 2023, como horizonte de 10 años con respecto a la finalización del trabajo para facilitar los cálculos de las proyecciones tanto de caudal como de carga para estos escenarios.

Se utilizaron caudales correspondientes al $85 \%$ de permanencia en el tiempo de la estación Juanchito $\left(133 \mathrm{~m}^{3 *} \mathrm{~s}^{-1}\right)$ y se tuvo en cuenta los vertimientos de contaminación puntual tales como industriales, municipales y zonas rurales. Para el sector doméstico municipal se consideró la cobertura de alcantarillado de los municipios, la eficiencia de las PTARs según su nivel de tratamiento (primario, secundario o terciario), criterios de operación y mantenimiento y el caudal tratado y no tratado. En la zona rural se asumió que no se tratan las aguas residuales porque la cobertura de tratamiento preliminar es casi nula. Igualmente, para todos los vertimientos se consideró la capacidad de autodepuración de los cuerpos hídricos confluentes al río Cauca.

\section{Construcción de las estrategias}

A partir de la revisión de literatura, se identificó la tendencia de las estrategias de control de contaminación por aguas residuales que se han aplicado tanto en la zona de estudio como a nivel mundial. En ese sentido se estructuró la estrategia centralizada y descentralizada de la siguiente manera.

\section{Estrategia centralizada (año 2023)}

Se proyectaron los caudales y cargas obtenidas en la línea base con las tasas de crecimiento determinadas por el Departamento Administrativo Nacional de Estadística (DANE, 2013) al 2023, con la intención de observar las variaciones en cuanto a caudal y carga, según el crecimiento de la población de las cabeceras municipales. La producción per cápita, los caudales y cargas de las aguas residuales generadas por el sector industrial se mantuvieron. A partir de esta información estimada, se planteó que las cabeceras municipales que descargan al río Cauca en la Cuenca Alta (sin priorización), tienen planta de tratamiento de aguas residuales en funcionamiento y las que se han construido posteriores al año base, cuentan con una eficiencia de remoción en $\mathrm{DBO}_{5}$ del $80 \%$ teniendo en cuenta lo que indicaba el Decreto 1594/1984 que regía durante el desarrollo de la investigación, actualmente este se encuentra derogado por la Resolución 0631 de 2015 (y por la serie de Compilación de Decretos Reglamentarios Únicos Decreto 1076 de 2015), la cual establece los parámetros y 
los valores límites máximos permisibles en los vertimientos puntuales en términos de concentraciones y no de eficiencias de remoción.

\section{Estrategia descentralizada (año 2023)}

Con el fin de priorizar las inversiones económicas, se definieron dentro de cada subcuenca las cabeceras municipales con mayor aporte de carga al río tributario al año 2023. Se construyó una estrategia que considerara la minimización y reúso, a la cual se le calcularon los volúmenes requeridos y producidos al aplicarse diferentes medidas dentro de la vivienda, como el uso de fuentes alternas de agua, uso de aparatos de bajo consumo y cambio de hábitos. Con la intención de calcular la disminución en el consumo y por ende en el caudal producido de aguas residuales municipales.

Se asumió que se recolectan y tratan las aguas residuales por la PTAR municipal cuyo efluente podría ser dispuesto para el riego de cultivos de caña de azúcar (cultivo predominante en la zona de estudio) con una superficie cultivada de 208121ha con base en la información obtenida de CVC (2013) y CRC (2013)). Estos cultivos de caña podrían estar en el entorno a las PTARs de todas las cabeceras municipales priorizadas. Por lo tanto, las áreas potenciales de riego del cultivo predominante dentro de la zona de estudio, fueron asociadas con el caudal de producción proveniente del efluente de la PTAR (modificado por las medidas de prevención y minimización consideradas para las viviendas). Estas áreas fueron a su vez revisadas utilizando un sistema de información geográfica-SIG, para identificar la disponibilidad de área real. A partir de esta relación se calculó la carga final vertida al río Cauca.

Para estimar el área de riego se realizó el balance entre la demanda y oferta del recurso hídrico, permitiendo identificar la existencia de déficit y/o excesos de agua. Para este cálculo, se efectuó un balance entre la demanda (evapotranspiración media mensual) y la oferta (precipitación media mensual). Se generaba déficit si la demanda superaba el aporte de la precipitación bajo el escenario considerado. Los datos de precipitación media mensual multianual y la evaporación media mensual multianual, se obtuvieron del Boletín Hidrológico de la CVC y del reporte de CENICAÑA del año (2004 al 2011), del cual se identificaron las estaciones pluviométricas cercanas a las cabeceras de los municipios (Jaramillo, 2014).

A partir del caudal de aguas residuales (estimados con la reducción que genera la minimización en la vivienda), se calculó el área potencial de riego. En este cálculo se tuvo en cuenta que las zonas de las cabeceras municipales priorizadas, tiene una pendiente favorecedora hacia el río, lo cual hace factible el riego por gravedad. Posteriormente para verificar que esta área potencial fuese realmente disponible, se superpuso con información geográfica (SIG), a través de la herramienta de ArcGis versión 10.1, encontrándose que el área potencial era igual que la real para cada uno de los casos.

\section{Comparación de carga producida y carga vertida}

Se compararon las cargas producidas y vertidas, obtenidas de las bases de datos de cada una de las estrategias (centralizada y descentralizada), construidas a partir de la línea base; mediante la estimación de las proporciones de reducción lograda por cada estrategia respecto al vertido en el tramo total al año base. Adicionalmente, se calculó la reducción total de la carga vertida con respecto a la producida en cada una de las estrategias.

\section{Niveles de OD y $\mathrm{DBO}_{5}$ en el tramo crítico. Aplicación del modelo QUAL2K}

Para comparar el efecto de las dos estrategias en la calidad del agua del río Cauca 
(centralizada y descentralizada), se evaluó el impacto en la calidad del agua del río Cauca a lo largo del tramo La Balsa - Anacaro. Para ello, se usó el modelo Qual2K versión 2.07, el cual ya estaba calibrado y verificado por Urrego (2014). Este modelo, es una herramienta que permite la modelación simultánea de cauce principal y tributarios (modelación dendrítica). La aplicación del modelo, incluyó el componente hidráulico (caudales) y la componente asociada a variables de calidad $\left(\mathrm{DBO}_{5}, \mathrm{OD}\right)$.

\section{Resultados y discusión Línea Base (2011)}

Las cuencas: Palo, Guachal, Tuluá y las cabeceras municipales: Cali y Buga, fueron los principales vertimientos en cuanto a carga $\mathrm{DBO}_{5}$ encontrados en el área de estudio, estos suman el $62 \%$ de la carga. Asimismo, dentro de la cuenca del río Palo, los municipios con mayor carga en $\mathrm{DBO}_{5}$ son Puerto Tejada, Toribio y Corinto. Los resultados son presentados en la Tabla 1 (Ver anexos).

\section{Estrategias aplicadas Centralizada}

La carga doméstica de las cabeceras municipales fue afectada por un porcentaje de remoción del $80 \%$ en $\mathrm{DBO}_{5}$ porque se construirán PTARs sin priorización. La carga doméstica de la zona rural nucleada perteneciente a esta área del Cauca fue proyectada al año 2023. La carga industrial se considera para el año 2023, igual que para el año base (2011), considerando que el crecimiento de la producción de carga contaminante sería reducido con estrategias de Producción Más Limpia-PML o con nuevos sistemas de tratamiento (Ver anexos, Tabla 1).

\section{Descentralizada}

Esta estrategia propone una combinación de sistemas centralizados con el fin de adaptar las tecnologías a las condiciones locales y de desarrollo de la zona de estudio. En los municipios priorizados a partir de la línea base (2011) (Ver Tabla 1), pertenecientes a las subcuencas con mayor aporte de carga contaminante en la cuenca alta del río Cauca, se asumió que se implementarían estrategias de minimización y reúso de agua a nivel de la vivienda, como se explica a continuación, cumpliéndose así los pasos 1 y 2 de la metodología de Nhapi \& Gijzen (2005):

A nivel de vivienda: se idealiza un escenario en el que haya cambio de hábitos (menor uso de agua potable) y uso aparatos de bajo consumo (para reducción en caudales) en viviendas existentes y proyectadas o nuevas. Sistemas de captación, tratamiento y conducción para el reúso de aguas grises y aprovechamiento de aguas Iluvias. Esto con base en el cumplimiento de la ley de uso eficiente del agua (Congreso de Colombia, 1997).

Para este caso de estudio, se consideraron dos tipos de vivienda: viviendas unifamiliares habitadas por 4 personas (DANE, 2005) y viviendas multifamiliares conformadas por 4 edificios de 15 apartamentos cada uno. Se asumió que el 70\% de la población para el año 2023 vivirá en viviendas unifamiliares y el $30 \%$ en viviendas multifamiliares (Zambrano, 2012).

En la (Tabla 2), son presentados los volúmenes considerados por cada uso con aparatos de bajo consumo. En cuanto a cambio de hábitos se consideró la reducción del tiempo en la ducha a 5 minutos, porque se encontró que una ducha de 5 min necesita $100 \mathrm{~L}$ con un cabezal normal y $50 \mathrm{~L}$ con una ducha ahorradora equipada de un reductor de caudal, contra 250L que consume una ducha que no tiene un sistema aspersor (Howard \& Bartram, 2003; ECODES, 2011; Fundación Aquae, 2016). 
Tabla 2. Estrategia descentralizada para el río Cauca. Demanda de agua por uso doméstico

\begin{tabular}{|c|c|c|c|c|}
\hline Uso del Agua & Especificaciones & $\begin{array}{l}\text { Consumo } \\
\text { Unitario (L) }\end{array}$ & Frecuencia & $\begin{array}{l}\text { Volumen (m3/ } \\
\text { vivienda*mes) }\end{array}$ \\
\hline Cocina & Lavado de utensilios & $10(b)$ & - & $1,20(b)$ \\
\hline \multirow{4}{*}{ Lavamanos } & Lavado de dientes & $1,3(a)$ & 3 veces* $d^{-1}(a)$ & 0,47 \\
\hline & Lavado de manos & $1,3(a)$ & 3 veces $* d^{-1}(a)$ & 0,47 \\
\hline & Lavado de cara & $1,95(a)$ & $1 v e z^{*} d^{-1}(a)$ & 0,23 \\
\hline & Afeitado & $1,95(a)$ & $1 v e z^{*} d^{-1}(a)$ & 0,23 \\
\hline Ducha & $\begin{array}{l}\text { Cambio de } \\
\text { hábito y aparato }\end{array}$ & $6,5(a)$ & $5 \mathrm{~min}$ & 3,90 \\
\hline Lavado de ropa & Lavadora & $150(b)$ & 3 veces*semana $^{-1}(b)$ & 1,80 \\
\hline Aseo & Pisos, baños, otros & $48(b)$ & 15días*mes ${ }^{-1}(a)$ & 0,72 \\
\hline Sanitario & $\begin{array}{l}\text { Inodoro de bajo } \\
\text { consumo }\end{array}$ & $2,3(b)$ & 5 descarga*d-1 $(a)$ & 1,38 \\
\hline $\begin{array}{l}\text { Riego en vivienda } \\
\text { unifamiliar }\end{array}$ & $\begin{array}{l}\text { Antejardín, patio, } \\
\text { lavado de carro }\end{array}$ & - & - & $2,00(b)$ \\
\hline $\begin{array}{l}\text { Riego de jardines } \\
\text { y aseo zonas } \\
\text { comunes en vivienda } \\
\text { multifamiliar }\end{array}$ & Zonas comunes & $9000(b)$ & $\begin{array}{l}\text { 1/15 1edificio } \\
\text { (15viviendas)(b) }\end{array}$ & $0,60(b)$ \\
\hline & & & TOTAL & 13,00 \\
\hline
\end{tabular}

Fuentes: (a) (EMASESA, 2013) (b) (Zambrano, 2012)

Cambiando las unidades del volumen mensual se obtiene una dotación L/hab/d, la cual para esta estrategia según el tipo de vivienda es: multifamiliar $87(\mathrm{~L} / \mathrm{hab} / \mathrm{d})$ y unifamiliar $98(\mathrm{~L} / \mathrm{hab} / \mathrm{d})$.

En la PTAR: los municipios que fueron priorizados en la línea base y que no contaban con una PTAR, fueron construidos hipotéticamente en los escenarios. Adicionalmente, se asumió que todas las plantas de los municipios priorizados removerían mínimo el $80 \%$ de la carga en $\mathrm{DBO}_{5}$, independientemente de las tecnologías de tratamiento que estas presentaban. Por ende, las que se encuentran en la realidad por debajo de esta eficiencia se asume en los escenarios, que estas ya optimizaron sus procesos.

Después de la PTAR: para el cálculo del balance oferta - demanda de agua, se efectuó un balance entre la demanda (evapotranspiración media mensual) y la oferta (precipitación media mensual). Estos datos fueron ingresados a la hoja de cálculo de Jaramillo, (2014), la cual a partir del caudal de aguas residuales (estimados con la reducción que genera la minimización en la vivienda), calcula el área potencial de riego. Los resultados de las áreas a regar son presentados en la (Tabla 3). 
Tabla 3. Área a regar con agua residual tratada

\begin{tabular}{|c|c|c|c|c|}
\hline Vertimiento & $\begin{array}{l}\text { Cabecera } \\
\text { Municipal }\end{array}$ & $\begin{array}{l}\text { Caudal de Agua } \\
\text { Residual }\left(m^{*} s^{-1}\right)\end{array}$ & $\begin{array}{l}\text { Área de Riego } \\
\text { Potencial (ha) }\end{array}$ & $\begin{array}{c}\text { Área de Riego } \\
\text { Real (ha) }\end{array}$ \\
\hline \multirow{3}{*}{ Río Palo } & Puerto Tejada & 0,03 & 79 & 79 \\
\hline & Toribio & 0,02 & 49 & 49 \\
\hline & Corinto & 0,02 & 88 & 88 \\
\hline Cali & Cali & 2,22 & 1982 & 1982 \\
\hline \multirow{4}{*}{ Río Guachal } & Palmira & 0,18 & 782 & 782 \\
\hline & Pradera & 0,03 & 138 & 138 \\
\hline & Candelaria & 0,01 & 36 & 36 \\
\hline & Florida & 0,04 & 168 & 168 \\
\hline Buga & Buga & 0,08 & 126 & 126 \\
\hline Río Tuluá & Tuluá & 0,14 & 349 & 349 \\
\hline
\end{tabular}

\section{Comparación de estrategias}

En la Tabla 1 (Anexos), se muestran tanto la condición de línea base (2011) como las bases de datos obtenidas en la construcción de ambas estrategias al año 2023. En esa tabla se puede observar, pese a que hay un incremento de las cargas producidas al 2023 debido al crecimiento de la población, las implementaciones de ambas estrategias reducen la carga final vertida; siendo la estrategia descentralizada la que genera una mayor reducción. Esta reducción corresponde al 63\%, mientras que con estrategia centralizada está reducción es solo del 54\%. La estrategia descentralizada logra una mayor reducción debido al manejo de agua residual desde la fuente de generación, disminuyendo la propagación de la contaminación debido al transporte de largas distancias de agua residual. Especialmente en los municipios con mayor carga contaminante aguas arriba del punto crítico (mínimo de OD).

Otros factores que influyeron en este resultado, es la reducción del consumo de agua potable en la estrategia descentralizada, ya que el reúso permitió cubrir usos en los que no se requiere esta calidad de agua. Por lo tanto, la generación de agua residual es mucho menor. Permitiendo afirmar que el sistema descentralizado no es solo una solución a largo plazo, sino que es más confiable en términos de impacto en la calidad de los recursos naturales y es más rentable.

\section{Aplicación del modelo Qual2K}

Los resultados del modelo descrito Qual2K y aplicado a lo largo del tramo La Balsa-Anacaro (Figura 4) (Anexo), permitieron evaluar el impacto acumulado de las estrategias centralizada y descentralizada y verificar la respuesta del río Cauca en su cuenca alta ante estas estrategias, dando cumplimiento al tercer paso de la metodología planteada por Nhapi \& Gijzen (2005).

La Figura 4 (Anexos), muestra que la estrategia descentralizada eleva los niveles de oxígeno en el punto crítico, a la altura de la estación Puerto Isaacs, aproximadamente a $3,5 \mathrm{mgOD}^{*} \mathrm{~L}^{-1}$, cuando la $\mathrm{DBO}_{5}$ en este punto es de aproximadamente $1,2 \mathrm{mg} \mathrm{DBO}_{5}{ }^{* \mathrm{~L}^{-1}}$; mientras que con la estrategia centralizada se alcanza aproximadamente a 2,5 mgOD* $\mathrm{L}^{-1}$, cuando la $\mathrm{DBO}_{5}$ en este 
punto es de aproximadamente $4,0 \mathrm{mg} \mathrm{DBO}_{5} * \mathrm{~L}^{-1}$. Se observa que el río obtiene una mejor respuesta ambiental con la estrategia descentralizada lo cual permitiría una mayor supervivencia de las especies en el punto crítico.

Los resultados presentados demuestran que sistemas de aguas residuales descentralizadas acompañados de la priorización de acuerdo al criterio mayor aportante de carga contaminante en cuanto a $\mathrm{DBO}_{5}$ (que a su vez podría influenciar a la priorización de los recursos económicos), es una estrategia que puede conducir a la gestión sostenible de aguas residuales en la cuenca alta del río Cauca, la cual va acorde a la regulación de prácticas de reutilización y a la definición de criterios de calidad lo cual facilita la articulación del marco conceptual con las acciones, haciendo viable la aplicación de la metodología de los Tres Pasos en un futuro, apoyados a su vez, en otras metodologías que promuevan la aceptación de estrategias descentralizadas y generen conciencia social para la protección del ambiente.

\section{Conclusiones}

La estrategia de los tres pasos, combinada con la priorización logran mayores remociones de carga contaminante comparada con la estrategia centralizada. Considerando el aporte de carga contaminante en el tramo de estudio de $169,54 \mathrm{~T} \mathrm{DBO}_{5} * \mathrm{~d}^{-1}$ (año base 2011), la estrategia centralizada (2023) que considera la construcción de PTARs a nivel de tratamiento secundario en todos los municipios de la cuenca redujo el aporte de carga contaminante a $86,34 \mathrm{~T} \mathrm{DBO}_{5} * \mathrm{~d}^{-1}$. Mientras que la aplicación de la estrategia descentralizada, también al año 2023 (considerando minimización, reúso, PTARs en cabeceras municipales y cuencas priorizadas) descarga al río Cauca una carga de solo $64,65 \mathrm{~T} \mathrm{DBO}_{5} * \mathrm{~d}^{-1}$ logrando reducciones de $54 \%$ y $63 \%$ respectivamente.
Para lograr una eficiente recuperación de la calidad del río Cauca en el tramo La BalsaAnacaro se requiere cambiar la estrategia convencional de "solución al final del tubo" por estrategias que involucren los conceptos de PML aplicadas al manejo del recurso hídrico: prevención y minimización, tratamiento para el reúso de los efluentes tratados en el sector agrícola, considerando la capacidad de autodepuración del cuerpo hídrico. Esto se evidenció en la modelación del parámetro de OD mínimo, puesto que para la opción centralizada (año 2023) se alcanzó un OD mínimo en el punto crítico del tramo La Balsa-Anacaro de 2,5 $\mathrm{mg}^{*} \mathrm{~L}^{-1}$, mientras que para la alternativa descentralizada el valor de OD mínimo simulado, para el año 2023 fue de $3,5 \mathrm{mg}^{*} \mathrm{~L}^{-1}$, en el punto crítico.

Los factores más influyentes que permitieron la reducción de carga afluente al río en el tramo La Balsa-Anacaro, son la eliminación del manejo de las aguas residuales tan cerca de la fuente de generación como fuese posible, reduciendo la propagación de la contaminación debido al transporte de agua residual a largas distancias.

La modelación utilizada, la cual incluye análisis dentrítico, es sumamente determinante para estudios de caso en los cuales se requiera evaluar el impacto acumulado de estrategias descentralizadas en un cuerpo hídrico principal. Por ende, se recomienda utilizar esta metodología en trabajos futuros.

\section{Agradecimientos}

Se agradece al Instituto de investigación Cinara de la Universidad del Valle por proporcionar los datos y gestionar otros adicionales con los cuales se hizo posible este estudio. Agradecemos, en especial al profesor Alberto Galvis por su dirección en la aplicación de esta metodología y al ingeniero Juan Gabriel Urrego quien realizó la modelación. Se agradece también al estudiante 
de doctorado Brayan Alexis Parra Orobio por el apoyo en la revisión del presente artículo.

\section{Literatura citada}

Chirisa, I., Bandauko, E., Matamanda, A., \& Mandisvika, G. (2017). Decentralized domestic wastewater systems in developing countries: The case study of harare (zimbabwe). Applied Water Science, 7(3), 1069-1078.

Congreso de Colombia. (1997). "Ley 373 de 1997. Programa para el uso eficiente y ahorro del agua." Congreso de Colombia, ed., Bogotá D.C., Colombia. Disponible en: http://www.alcaldiabogota.gov.co/sisjur/normas/Norma1.jsp?i=342.

CRC (2013). Compilado de datos proporcionados por funcionarios de la CRC. Corporación Autónoma Regional del Cauca. Formato Excel. Información privada.

CRC (2010). Caracterización ambiental. Plan departamental de agua y saneamiento básico. CRC. Popayán.

CVC (2013). Compilado de datos proporcionados por funcionarios de la CVC. Corporación Autónoma Regional del Valle del Cauca. Formato Excel. Información privada.

CVC (2010). Boletín Hidroclimatológico. Red de Hidroclimatología. Dirección Técnica Ambiental.

DANE. (2005). Boletín Censo General. Bogotá, CoIombia. Disponible en: http://www.dane.gov.co/ index.php/estadisticas-por-tema/demografia-ypoblacion/censo-general-2005-1

DANE. (2013). Estimación y proyección de población nacional, departamental y municipal total por área 1985-2020. Consulta: junio 2013. Página web: http://www.dane.gov.co/index.php/poblacion-y-demografia/proyecciones-de-poblacion.

Dou, T., Troesch, S., Petitjean, A., Gábor, P.T., \& Esser, D. (2017). Wastewater and rainwater management in urban areas: A role for constructed wetlands. Procedia Environmental Sciences, 37, 535-541. doi: https://doi.org/10.1016/j. proenv.2017.03.036

ECODES. (2011). Consejos prácticos para ahorrar agua Zaragoza, España. Ecología y DesarroIlo, ECODES. Consulta: noviembre 2012. Página web: http://www.consumoresponsable.org/actua/agua/agua_consejos_hogar.
EMASESA. (2013). Cálculo del consumo individual de agua. Empresa Metropolitana de Abastecimiento y Saneamiento de Aguas de Sevilla, S.A de España -(EMASESA). Consulta: noviembre 2013. Página web: http://www.aguasdesevilla. com/infantil/calculo/consumo.swf.

EMCALI (2012). Información proporcionada por funcionarios de EMCALI. Formato Excel. Información privada.

Fundación Aquae. (2016). Cuánta agua de consume en la ducha por minuto. Consultada en: 02, 2018. Página web: https://www.fundacionaquae. org/blog/infografias/cuanta-agua-consume-laducha-minuto/

Gupta, N. (2016). Centralised v/s decentralised wastewater treatment solutions - a case study. IJSRD - International Journal for Scientific Research and Development, 4(1), 1019-1024.

Howard, G., \& Bartram, J. (2003). La cantidad de agua domiciliaria, el nivel del servicio y la salud. WHO Document Production Services.

Jaramillo, M. F. (2014). Potencial de reúso de agua residual doméstica como estrategia para el control de la contaminación por aguas residuales en el Valle geográfico del río Cauca. Escuela de Recursos Naturales y del Medio Ambiente. Universidad del Valle. Tesis de Maestría. Cali, Colombia.

Moreno A., G.A. (2014). Análisis ambiental de la cuenca alta del río Cauca, sus actores principales y la afectación en el suministro de agua potable para la ciudad de Santiago de Cali. ¿Qué acción tomar? Universidad ICESI. Tesis de Maestría. Cali, Colombia.

Nhapi, I., \& Gijzen, H. (2005). A 3-step strategic approach to sustainable wastewater management. Water SA, 31(1).

Polanía J., A.V. (2014). Comparación de dos tipos de estrategias para el control de la contaminación por aguas residuales en la cuenca alta del río cauca. Tesis de Pregrado. Universidad del Valle. Cali, Colombia.

Sánchez, F. (2013). Costos detallados de algunas PTARs diseñadas y construídas por Sánchez Ingeniería en el Valle del Cauca. Sánchez Ingeniería. Formato Excel. Información privada. 
Sandoval, M., \& Ramírez, C. (2007). El río Cauca en su valle alto. Un aporte al conocimiento de uno de los ríos más importantes de Colombia. CINARA-CVC.

SSPD (2010). Informe técnico sobre sistemas de tratamiento de aguas residuales en Colombia. Línea base 2010. Superintendencia de Servicios Públicos Domiciliarios. Bogotá.

SSPD (2013a). Reporte de alcantarillado. TécnicoOperativo. Plantas de tratamiento de Aguas Residuales. Sistema Único de Información de Servicios Públicos - SUI. Consulta: febrero 2013. Página web: http://www.sui.gov.co/.

SSPD (2013b). Informe técnico sobre sistemas de tratamiento de aguas residuales en Colombia. Consulta: febrero 2018.

Suarez, C. (2010). Tratamiento de aguas residuales municipales en el Valle del Cauca. Escuela de
Ingeniería de Recursos Naturales y del Ambiente. Universidad del Valle. Maestría en Ingeniería Área de Énfasis en Ingeniería Sanitaria y Ambiental. Cali.

Urrego, J.G. (2014). Estudio de la capacidad de autodepuración del río Cauca en el tramo Salvajina - Anacaro utilizando modelación dendrítica de flujo permanente. (Tesis de Pregrado), Universidad del Valle, Cali, Colombia.

Zambrano, D. (2012). Minimización y prevención como estrategia para el control de la contaminación por aguas residuales municipales en la zona de expansión de Cali. (Tesis de maestría), Universidad del Valle, Cali, Colombia.

Zorrilla, Y. (2012). Aspectos económicos en el control de la contaminación por aguas residuales en el contexto de la gestión integrada del recurso hídrico. Universidad del Valle. Cali, Colombia. 